\title{
Informe bibliográfico sobre la lógica (epistémica) de la conciencia
}

\author{
Bibliographic Report on (Epistemic) \\ Awareness Logic
}

\author{
CLAUDIA FERNÁNDEZ-FERNÁNDEZ \\ UNIVERSIDAD DE MÁLAGA
}

Recibido: 05/11/2019 Aceptado:11/11/2019

\section{RESUMEN}

La lógica de la conciencia es una extensión de la lógica epistémica que solventa el problema de la omnisciencia lógica incorporando un operador de conciencia para separar el conocimiento explícito del implícito. Este informe recopila los principales textos tanto de los orígenes de esta lógica, así como de sus desarrollos en las últimas tres décadas. En concreto analiza los enfoques desde la lógica epistémica dinámica, desde su combinación con otras lógicas y los enfoques de teoría de juegos.

\section{PALABRAS CLAVE \\ LÓGICA EPISTÉMICA - CONOCIMIENTO EXPLÍCITO - CONCIENCIA - ACCIÓN EPISTÉMICA}

\begin{abstract}
Awareness Logic is an extension of epistemic logic that solves the problem of logical omniscience by incorporating an awareness operator that separates the explicit knowledge from the implicit one. This report collects the most prominent works regarding the beginnings of this logic, as well as its developments in the past three decades. Specifically, it reviews the approaches from Dynamic Epistemic Logic, from the ones that combine other logics with Awareness Logic and those from Game Theory.

KEYWORDS

EPISTEMIC LOGIC - EXPLICIT KNOWLEDGE - AWARENESS EPISTEMIC ACTION
\end{abstract}

(C) Contrastes. Revista Internacional de Filosofía, vol. XXIV-Nº3 (2019), pp. 105-120. ISSN: 1136-4076

Departamento de Filosofía, Universidad de Málaga, Facultad de Filosofía y Letras Campus de Teatinos, E-29071 Málaga (España) 
EL PRESENTE INFORME ESTÁ ENFOCADO a proporcionar una visión general de la bibliografía más relevante sobre la lógica (epistémica) de la conciencia. ${ }^{1}$ Para poder analizar dicha bibliografía es conveniente realizar un recorrido cronológico. Comenzaremos con los antecedentes lógicos de la lógica de la conciencia, asumiendo como conocimiento previo la base teórica de la lógica epistémica. Posteriormente analizaremos los distintos desarrollos que ha tenido esta lógica, con especial énfasis en aquellos relacionados propiamente con la lógica epistémica en el siglo XXI.

Antes de entrar en materia es conveniente hacer una aclaración terminológica: el término «conciencia» ${ }^{2}$ es la traducción del vocablo inglés awareness, por lo que cabría entenderlo más bien como sinónimo de «percatarse» o «tener en cuenta», pues se trata, en efecto, de una «conciencia», pero exclusivamente epistémica, es decir, referida al conocimiento (proposicional). Por tanto, la lógica (epistémica) de la conciencia es una lógica epistémica (esto es, del conocimiento) que incorpora la conciencia del agente, en tanto que este tiene la capacidad de percatarse de aquella información que conoce, para diferenciar así el conocimiento (o la creencia) real (explícito) del potencial (implícito).

\section{ANTECEDENTES LÓGICOS Y CREACIÓN DE LA LÓGICA DE LA CONCIENCIA}

No es posible hablar de lógica de la conciencia sin hablar previamente de su origen: el problema de la omnisciencia lógica en la lógica epistémica. Muy brevemente se podría resumir esta cuestión explicando el hecho de que, en la lógica epistémica tradicional, iniciada como tal en (Hintikka, 1962), ${ }^{3}$ los agentes epistémicos conocen necesariamente todas las consecuencias lógicas de su conocimiento, por tanto, son omniscientes lógicos. Para solucionar este problema se han propuesto un amplio número de estrategias. Una gran parte de estas estrategias distinguen entre un conocimiento implícito, siendo este coincidente con el tradicional de la lógica epistémica, y, por tanto, omnisciente; y un conocimiento explícito, referido a aquello que el agente realmente sabe, por tanto, más restringido que el anterior. Los procedimientos por los cuales se realiza esta distinción son muy diversos, teniendo todos ellos en común

1 Cabe resaltar que la totalidad de la bibliografía sobre el tema está redactada en inglés, pese a la gran variedad de orígenes de sus autores. Una búsqueda exhaustiva de bibliografía específica en castellano ha resultado infructuosa.

2 No distinguimos entre «conciencia» y «consciencia», aunque esta diferencia haya suscitado acalorados debates filosóficos, en este contexto se hace innecesaria.

3 Aunque la idea original aparece una década antes en (von Wright, 1951). 
la búsqueda de representación del conocimiento de agentes con recursos limitados, esto es, agentes epistémicos más similares a los seres humanos o a máquinas de computar.

Dentro de las estrategias que realizan esta división se distinguen, a grandes rasgos, dos tipos: las (a) sintácticas y las (b) semánticas. La diferencia principal radica en qué conocimiento se toma como punto de partida del sistema y, a partir de ahí, de qué modo se obtiene el conocimiento restante. Así las estrategias (a) sintácticas establecen el conocimiento explícito como punto de partida (definido como un listado arbitrario de fórmulas o proposiciones) y de ahí se extrae el conocimiento implícito, en la mayoría de los casos a través de procesos deductivos. Frente a esto, las estrategias (b) semánticas toman la lógica epistémica y su semántica de mundos posibles como base, es decir, el conocimiento implícito, y elaboran métodos para limitar ese conocimiento a lo realmente conocido por el agente, es decir, a lo explícito.

Una de estas estrategias semánticas es la lógica de la conciencia, en tanto que propone introducir la conciencia en la estructura de la lógica epistémica para que actúe a modo de filtro, seleccionando así el conocimiento explícito a partir del implícito que coincida con la conciencia del agente. Obviamos aquí los detalles técnicos de la propuesta, aunque se hace necesario resaltar que la conciencia que aquí se propone es vista desde un punto de vista sintáctico ${ }^{4}$, es decir, como un listado arbitrario de fórmulas. Siendo esto así, se puede explicar esta conciencia como un saco de fórmulas que, al superponerse con las fórmulas del conocimiento implícito, da como resultado el conocimiento explícito.

Aunque el problema de la omnisciencia lógica ya lo menciona el propio creador de la lógica epistémica, por ejemplo, en (Hintikka, 1975), proponiendo aquí además una posible solución (los mundos imposibles), no será hasta finales de los años ochenta cuando aparezca la lógica de la conciencia, experimentando a partir de su creación un notable crecimiento. La propuesta inicial está recogida en (Fagin y Halpern, 1988), un artículo que supone la reedición de un texto del año 1985 presentado en la novena edición del International Joint Conference on Artificial Intelligence en Los Ángeles, EE. UU. Además de proponer las primeras versiones de esta lógica, la Logic of Awareness y Logic of General Awareness, en los apartados 4 y 5 respectivamente, los autores realizan previamente un resumen del estado de la cuestión con bastante profundidad técnica, así como teórica, haciendo hincapié en los errores que, según ellos, se han cometido en las soluciones

4 Por este motivo a la lógica de la conciencia se la denomina una estrategia mixta semántica-sintáctica, pues es en sí una estrategia semántica, pero incorpora un elemento puramente sintáctico. 
propuestas hasta la fecha para solventar el problema de la omnisciencia. El error que destacan en primer lugar, y se convertirá en central, es la falta del uso de una noción de «conciencia» en los planteamientos epistémicos, es decir, el hecho de haber pretendido formalizar el conocimiento de agentes epistémicos sin tener en cuenta la necesidad de que han de ser conscientes de aquello que conocen. ${ }^{5}$ Será esta la máxima que inspire la creación de la lógica de la conciencia, así como sus futuros desarrollos.

\section{Desarrollos de la lóGicA DE la CONCIENCIA HASTA El SIGlo XXI}

La primera revisión crítica que se conoce es (Konolige, 1986), justo un año después de la presentación de la lógica de la conciencia en el congreso indicado más arriba del año 1985. Konolige, que aboga por un enfoque puramente sintáctico de la lógica epistémica, criticará el empleo insistente de la semántica de Kripke (o de mundos posible) en toda la lógica epistémica $\mathrm{y}$, en concreto, en la lógica de la conciencia, y lo contrapondrá a su modelo deductivo sintáctico, mostrando las ventajas de este último.

Durante la década de los noventa no se encuentran muchos trabajos específicos sobre el tema, más allá de los que realizan los propios creadores de esta lógica. Destaca, aun así, el artículo (Huang y Kwast, 1990) como trabajo que menciona y estudia la lógica de la conciencia, pero en aras de incorporarla a otros sistemas o planteamientos que los autores se proponen desarrollar. En concreto, este trabajo utiliza la noción de conciencia para definir nuevas formas de negación y de implicación. Sin embargo, en esta década los creadores de la lógica de la conciencia, junto con otros dos investigadores (Moses, Y. y Vardi M. Y.), publicaron el libro (Fagin et. al., 1995). Este libro, titulado «Razonando sobre el Conocimiento» (del inglés Reasoning about Knowledge), supuso una importante recopilación de los avances en lógica epistémica hasta la fecha ${ }^{6}$ y recoge los principales resultados metateóricos, así como las distintas formalizaciones del conocimiento común y distribuido en sistemas multi-agentes.

A su vez, en su capítulo noveno, titulado Logical Omniscience, los autores clasifican y explican las distintas estrategias para evitar la omnisciencia lógica,

5 Como dirán los autores, en una obra posterior, refiriéndose a la creación de la lógica de la conciencia: «The underlying idea is that it is necessary to be aware of a concept before one can have beliefs about it. One cannot know something of which one is unaware». (Fagin et. al., 1995, p. 337. Cursivas del original.)

6 Ese mismo año se publicó también el libro (Meyer y van der Hoek, 1995), que junto con (Fagin et. al., 1995) conforman la bibliografía básica y más relevante sobre la lógica epistémica de la década de los noventa. En el libro de Meyer y van der Hoek se menciona la lógica de la conciencia de Fagin y Halpern en el apartado 2.8, realizando un breve resumen de los puntos más importantes de esta. 
situando a la lógica de la conciencia como una estrategia mixta semánticasintáctica. Será en este punto donde desarrollen con algo más de detalle lo ya planteado en el año 1988 y añadan al final del citado capítulo diversas vías para los desarrollos futuros de esta lógica. En concreto, dejan abierta la posibilidad de imponer algún tipo de restricciones adicionales al conjunto de conciencia (por ejemplo, estar cerrado bajo subfórmulas o formado exclusivamente por proposiciones atómicas). Serán estas posibilidades las que propicien el rápido crecimiento de esta lógica en los años venideros.

Si bien se publicaron algunos trabajos que mencionaban a modo de recopilación la lógica de la conciencia, hasta donde nos consta no hay otro estudio de la década de los noventa que convenga resaltar aquí. Sin embargo, con la llegada del nuevo siglo sí se puede afirmar que comienza el rápido crecimiento que venimos anunciando. Por este mismo motivo, y dado que la mayoría de los desarrollos se superponen en el tiempo, a partir de este punto conviene dejar a un lado el orden cronológico, para establecer una clasificación temática y procedimental de los desarrollos de la lógica de la conciencia.

\section{Desarrollos de la lóGica de la conciencia en el Siglo XXI}

En lo que sigue, vamos a agrupar la mayoría de los trabajos recopilados que incorporan, modifican o revisan la lógica de la conciencia en los siguientes tres apartados, en función del tipo de lógica que emplean (enfoque lógico) y/o de sus aplicaciones prácticas (implementaciones lógicas): (1) enfoque de lógica epistémica (y dinámica), (2) enfoques combinados con otras lógicas y (3) enfoque de teoría de juegos y aplicaciones a la economía.

\section{III.1. ENFOQUE DE LÓGICA EPISTÉMICA (Y DINÁMICA)}

Comenzamos por el enfoque sobre la conciencia desde la lógica epistémica dinámica ${ }^{7}$ (LED, de aquí en adelante). Si la lógica epistémica es la formalización del conocimiento, la LED es la representación formal de los flujos de información, entendiendo estos como cualquier proceso comunicativo en el que se producen intercambios y transformaciones de informaciones de diversa índole.

En cuanto a los intercambios de la información bastaría con emplear la ya conocida lógica epistémica en su versión multi-agente, pues permite formalizar una gran variedad de argumentos deductivos (sobre todo si se emplea también la vertiente dedicada a la creencia, la lógica doxástica). Sin embargo, en lo que atañe a las transformaciones de las informaciones, la lógica epistémica, pese

7 Ver (Murillo-Corchado y Nepomuceno-Fernández, 2019) para una breve revisión sobre los orígenes de esta denominación en castellano y (van Ditmarsch et. al., 2008) y (van Benthem, 2011) como los dos principales manuales (en inglés) sobre el tema. 
a sus múltiples extensiones, carece de recursos para poder formalizarlos, pues realiza siempre una representación estática del conocimiento, es decir, sólo puede mostrar un estado epistémico concreto. Para poder captar formalmente el proceso dinámico que experimenta la información en la comunicación surge la LED. ${ }^{8}$

El concepto de LED hay que entenderlo como un término hiperónimo e interdisciplinar que, en palabras de Murillo-Corchado y NepomucenoFernández, «se ha beneficiado de la confluencia de esfuerzos por parte de investigadores cuyo trabajo se realiza en diversas disciplinas, principalmente, filosofía, lingüística, álgebra, ciencias de la computación y teoría de juegos, entre otras, lo que le da un marcado sello de interdisciplinariedad» (MurilloCorchado y Nepomuceno-Fernández, 2019, p. 74).

LED, como hiperónimo, incluye, entre otras, la tradición lógica del anuncio público (Public Announcement), así como del sistema $A G M$ (nombrado por sus creadores, Alchourrón, Gärdenfors y Makinson) y el de revisión de creencias (Belief Revision) y se ha seguido desarrollando por muchas vertientes lógicas para captar cada vez más sutilezas de la información que comparten los agentes no omniscientes, es decir, con recursos limitados. Probablemente la noción clave de toda LED sea la de acción epistémica. Se entiende por acción epistémica un cambio (positivo o negativo) en la información disponible para el agente. Formalmente se representa mediante un cambio en el modelo semántico (tradicionalmente de mundos posibles, aunque se han explorado otros, como los modelos de vecindad) incorporando o eliminando alguna información (o bien una fórmula completa o bien proposiciones atómicas, en función de las definiciones de cada sistema) de una parte del modelo.

En las acciones de conciencia, que se pueden englobar bajo los términos de volverse consciente y volverse inconsciente, se ve afectado el conjunto de conciencia del modelo, añadiendo o extrayendo, respectivamente, alguna información. Los cambios en la conciencia repercuten normalmente en el conocimiento explícito (o real) del agente, el cual en los sistemas con conciencia depende directamente de esta. Estas acciones, no obstante, han sido nombradas con un amplio elenco de términos en función de la tradición que siguieran los autores o las especificidades del sistema en el que se incorporasen. Así, por ejemplo, (van Benthem, 2011) dedica el quinto capítulo de este manual a la «dinámica de la inferencia y la conciencia» (Dynamics of inference and awareness), y menciona las acciones de «darse cuenta» (acts of realization), nombre bajo el cual engloba diversas acciones que amplían el conjunto de conocimiento explícito, y, por ende, el de la conciencia, del

8 Los motivos del surgimiento de LED son bastante más complejos. No obstante, en lo que respecta exclusivamente a la lógica epistémica y la comunicación, cabría mantener esta afirmación. 
agente. En general, la mayoría de los enfoques han optado por los términos access (referido al acceso a la información) o entertainment (en referencia a considerar una determinada información).

Los trabajos que incorporan, como elemento principal o, en su defecto, con bastante importancia dentro del estudio, las acciones de conciencia son muchos y no es posible revisarlos todos, por lo que mencionaremos algunos de ellos, dejando a muchos otros inevitablemente en el tintero. En cuanto a los autores más prolíficos en el ámbito de la lógica de la conciencia dinámica, más allá de sus propios creadores (y sus discípulos y colaboradores), se pueden resaltar en primer lugar a dos grandes figuras: Hans van Ditmarsch, ${ }^{9,10}$ quien, además, forma parte de los inicios de la lógica del anuncio público y también destaca en la rama de revisión de creencias; y Johan van Benthem, ${ }^{11}$ considerado por muchos uno de los «creadores» del giro dinámico en la lógica epistémica. Aunque hoy en día ya no se puede hablar de «escuelas» y «discípulos» con las acepciones clásicas, sí se distinguen dos grandes tradiciones de las que estos investigadores forman parte, aunque se entremezclen e inserten siempre en ámbitos de estudio más amplios e interdisciplinares. En calidad de «discípulos» de estos grandes investigadores, aunque el elenco es muy amplio, en referencia a los trabajos del ámbito de la lógica de la conciencia, es menester mencionar en la línea del primero a Tim French ${ }^{12}$ y del segundo a Fernando R. Velázquez-Quesada. ${ }^{13}$

En relación con las colaboraciones entre los primeros resaltan los trabajos (van Ditmarsch y French, 2009) y (van Ditmarsch y French, 2011). En el primero estudian los diversos cambios en la conciencia aplicada no sólo a fórmulas (proposiciones atómicas, en este caso), sino también a otros agentes dentro del grupo. Estos autores definen, a su vez, una operación para olvidar (eliminar de la conciencia) muy interesante. En el segundo trabajo, del año 2011, extienden la noción de awareness bisimulation, introducida ya en el trabajo anterior, para mostrar las relaciones entre diversos modelos y formalizar los cambios en la conciencia de los agentes. Distinguen aquí, además, entre volverse consciente o bien de todas las variables proposicionales de una

9 Para más información sobre este investigador ver: https://sites.google.com/site/hansvanditmarsch/.

10 El manual (van Ditmarsch et. al., 2008) se considera de lectura obligatoria hoy en día y engloba gran parte de las ramas de la LED.

11 Para más información sobre este investigador ver: https://staff.fnwi.uva.nl/j.vanbenthem/ .

12 Para más información sobre este investigador ver: https://research-repository.uwa. edu.au/en/persons/tim-french .

13 Para más información sobre este investigador ver: https://staff.fnwi.uva.nl/ f.r.velazquezquesada/lang-en/index.html . 
fórmula o bien de sólo una proposición en concreto. Por último, como trabajo conjunto con más investigadores, conviene resaltar (van Ditmarsch et. al., 2013), ${ }^{14}$ un artículo en el que recopilan parte de los avances mencionados en los anteriores y añaden la noción de conocimiento especulativo a una nueva lógica, presentando aquí su sistema axiomático. Los autores mantienen las acciones de cambios en la conciencia ya planteadas en los trabajos anteriores $y$, en general, se trata de un texto muy completo y accesible, a la vez que breve. Estos mismos autores han publicado recientemente (van Ditmarsch, et. al., 2018), un texto más extenso que, además de recopilar y extender los trabajos anteriores, cuenta con una amplia sección introductoria y una bibliografía muy completa, que puede servir como introducción a la actualidad de los cambios en conciencia dentro de sistemas de LED multi-agentes con bisimulación.

Con respecto a lo que se podría denominar la escuela de van Benthem, que incluye, en definitiva, a todo el centro de investigación ILLC, de la Universidad de Ámsterdam (Países Bajos), resaltamos aquí al investigador F. R. Velázquez-Quesada. En su tesis doctoral (Velázquez-Quesada, 2011), dirigida por van Benthem, reúne diversas maneras de representar la dinámica de la información atendiendo siempre a los pequeños detalles, es decir, siendo lo más minucioso posible para formalizar las múltiples sutilezas de la información. Probablemente el artículo más relevante con respecto a la conciencia que publicaron van Benthem y Velázquez-Quesada durante el desarrollo de esta tesis sea (van Benthem y Velázquez-Quesada, 2010). Se trata de un texto en el que recopilan las bases de la LED añadiendo un factor clave: la consideración de la inferencia (deductiva) también como acción epistémica, en cuanto que modifica el conocimiento explícito (y, en potencia, el implícito) del agente. A su vez definen las acciones que amplían o contraen el conjunto de conciencia, denominándolas en este caso add (añadir) y drop (soltar). Como dicen los autores en el resumen inicial de este texto, su «contribución puede ser vista como una "dinamización" de las lógicas de la conciencia existentes». ${ }^{15}$

En (Velázquez-Quesada, 2011) aparece ya el germen de una distinción fundamental, que ocupará gran parte de los futuros trabajos de este investigador: la diferencia entre conciencia-de (awareness of) y conciencia-de-que (awareness that). Esta distinción se engloba dentro del análisis del conjunto de informaciones disponibles para un agente epistémico no omnisciente, es decir, el prototipo de agente en LED. Velázquez-Quesada analiza las diversas

14 En la misma línea se publicó un año antes (van Ditmarsch, et. al., 2012).

15 Del inglés: «contribution can be seen as a 'dynamification' of existing awareness logics», (van Benthem y Velázquez-Quesada, 2010, p. 5). En esta misma línea se plantea el texto (Hill, 2010) que, por motivos de espacio, no revisaremos aquí. 
formas en que se ha formalizado la conciencia epistémica y presenta, en el cuarto capítulo, una combinación de ellas en las que distingue, por un lado, la conciencia-de, definida como el lenguaje que el agente tiene a su disposición, el conjunto de informaciones que considera en un mundo (estado epistémico) determinado; y por otro lado, lo que hoy se puede denominar conciencia-de-que, referida a aquella información que el agente ha reconocido como verdadera, es decir, aquello que sabe. Es importante no confundir esta definición de conciencia-de-que con la de conocimiento explícito, pues en el sistema que plantea el autor, para tener conocimiento (ya sea explícito o implícito) de una información, siempre es requisito indispensable que forme parte de la conciencia-de y sólo podrá ser explícito si el agente, además, es consciente-de-que es el caso.

Estos términos teóricos de conciencia-de y conciencia-de-que han sido renombrados de diversas formas en cada uno de los trabajos que los incorporan. Así, por ejemplo, en el sistema que se plantea en el cuarto capítulo de esta tesis las fórmulas que definen la conciencia-de se denominan «fórmulas de disponibilidad» (availability formulas), mientras que las que representan la conciencia-de-que son las «fórmulas de reconocimiento» (acknowledgement formulas). A nivel semántico, para cada mundo posible, las primeras se definen mediante una función proposicional de disponibilidad, mientras que las segundas vienen dadas por un conjunto de acceso que incluye lo que se ha reconocido como verdadero. El trabajo (Grossi y Velázquez-Quesada, 2010) incorpora este mismo sistema y destaca, además, por la insistencia en la «minuciosidad» de los anuncios (fine-grained announcements), esto es, por el estudio detallado de las sutilezas específicas de cada tipo de comunicación. Unos años después el texto (Grossi y Velázquez-Quesada, 2015) recopila una serie de ideas de trabajos anteriores, a la vez que supone el primer texto ${ }^{16}$ que incorpora con estos términos las nociones de «conciencia-de» (awareness of) y «conciencia-de-que» (awareness that). Los autores definen aquí el concepto central de conocimiento explícito como la conjunción de las dos conciencias, aunque de un modo algo idealizado, es decir, manteniendo cierto nivel de omnisciencia. Por último, y en esta misma línea, se puede resaltar el texto (Velázquez-Quesada, 2014) donde el autor además de recoger todos los avances anteriores, renombrando de nuevo algunos tipos de informaciones y acciones, plantea algunas acciones epistémicas ya estudiadas en referencia a la conciencia, pero aplicándolas ahora a otros sistemas, por ejemplo, a la revisión de creencias o los modelos de plausibilidad.

16 Así lo indican los autores, afirmando que «the paper develops the first approach that combines both notions of awareness, 'awareness of' and 'awareness that', thus allowing a more realistic representation of non-ideal agents» (Grossi y Velázquez-Quesada, 2015, p. 4072). 
Como continuación de esta línea de trabajo, a saber, la distinción entre los dos tipos de conciencia, se pueden mencionar los trabajos recientes (Fernández-Fernández y Velázquez-Quesada, 2019a) y (Fernández-Fernández y Velázquez-Quesada, 2019b), que plantean una revisión en profundidad de los fundamentos teóricos que subyacen a la lógica de la conciencia ${ }^{17} \mathrm{y}$, basándose en el resultado de esta revisión, definen un nuevo modelo lógico para la definición del conocimiento explícito con base en la combinación de las dos conciencias y empleando una estructura semántica basada en los modelos de vecindad. ${ }^{18}$

Para finalizar esta revisión de la lógica de la conciencia epistémica se debe hacer referencia a las recopilaciones publicadas en la última década sobre esta lógica. La más importante es probablemente la edición especial del Journal of Logic, Language and Information titulada Formal Models of Awareness, que vio la luz en el año 2014 (Ågnotes y Alechina, 2014). A su vez, cabe destacar que el Handbook of Epistemic Logic del año 2015 incluye un capítulo dedicado en exclusiva a la conciencia en la lógica epistémica (Schipper, 2015).

\section{III.2. ENFOQUES COMBINADOS CON OTRAS LÓGICAS}

En segundo lugar, conviene resaltar aquellos trabajos en los que la noción de conciencia se ha introducido en otras lógicas, dando lugar a nuevos sistemas lógicos que merecen atención. Con respecto a la combinación del operador de conciencia con la lógica de la justificación ${ }^{19}$, cabe mencionar, en primer lugar, el artículo (Sedlár, 2013). Este texto plantea un sistema en el que se combinan los operadores de la lógica de la justificación, a saber, términos de justificación y fórmulas de justificación, con la acción epistémica de volverse consciente, en este caso, referida a un grupo entero de agentes. Incorpora así a los modelos de justificación de grupos en un entorno multi-agente de justificación un tipo de conciencia introspectiva. En segundo lugar, el propio creador de la lógica de la justificación ha publicado recientemente (Artemov, 2018), un artículo en el que presenta un modelo conjunto de justificación y conciencia con el que plantea mejorar la formalización de un conocido ejemplo de B. Russell (Prime Minister Scenario) que, hasta la fecha, no se había estudiado desde este punto de vista epistémico. Los modelos de conciencia y justificación que propone

17 Tarea esta iniciada ya en (Fernández-Fernández y Velázquez-Quesada, 2018) a nivel teórico.

18 Estrategia similar a la que sigue el texto (Velázquez-Quesada, 2013), aunque sin la noción de conciencia.

19 Para más información sobre la lógica de la justificación ver (Artemov y Nogina, 2005) y (Artemov, 2008). 
el autor en este texto tienen un alto grado de complejidad técnica, motivo este que le proporciona una mayor precisión a la hora de captar las sutilezas de diversas situaciones epistémicas más cotidianas.

Como ejemplo de un trabajo muy completo, que implementa una gran variedad de temáticas epistémicas como son la lógica dinámica epistémica, la lógica de la justificación, la revisión de creencias y la lógica de la conciencia, cabe destacar (Baltag, et. al., 2014). Tal y como lo presentan sus autores en el resumen inicial, este trabajo incluye «una lógica completa y decidible para razonar sobre una noción de evidencia completamente fiable ("concluyente") y sus conexiones con el conocimiento y la creencia justificables (implícito/ as), así como con sus justificaciones explícitas». ${ }^{20}$ Más adelante estos autores mencionan específicamente la lógica de la conciencia y cómo emplean recursos de esta para definir su versión de conocimiento explícito justificado. Otro texto en la línea del anterior es (Burrieza y Yuste-Ginel, 2019), donde los autores introducen ambos tipos de conciencia (disponibilidad y reconocimiento) en su sistema para la evaluación de argumentos (estudios de preferencia entre argumentos) combinado con elementos de la lógica de la justificación. En concreto plantean un operador de disponibilidad y otro de reconocimiento, aplicado no sólo a fórmulas sino al resto de elementos de su amplio lenguaje, como, por ejemplo, argumentos.

\section{III.3. ENFOQUE DE TEORÍA DE JUEGOS Y APLICACIONES A LA ECONOMÍA}

En última instancia planteamos este enfoque, que se relaciona con la teoría de juegos (Game Theory), destacando lo que se conoce como teoría de la decisión (Decision Theory), que emplea técnicas de la teoría de modelos. A grandes rasgos, y obviando los complejos tecnicismos matemáticos que conlleva, se puede decir que este planteamiento implementa las bases de la lógica de la conciencia en una estructura lógica distinta, manteniendo sus fundamentos teóricos y permitiendo su aplicación a otros campos, tradicionalmente más alejados de la lógica epistémica en su interpretación más filosófica.

Este desarrollo de la lógica de la conciencia ha sido muy fructífero en sus aplicaciones a la teoría económica, en concreto a diversos tipos de actividades financieras especulativas. Así, por ejemplo, (Heifetz et. al., 2006) implementa su propuesta en diversos casos prácticos de transacciones económicas representando el conocimiento de los agentes comerciales. Destaca sobre todo el estudio no sólo de la conciencia (awareness), sino también de la

20 Traducción propia de «a complete, decidable logic for reasoning about a notion of completely trustworthy ('conclusive') evidence and its relations to justifiable (implicit) belief and knowledge, as well as to their explicit justifications» (Baltag, et. al., 2014, p. 49). 
inconciencia (unawareness), en referencia a los procesos de negociación donde cada uno de los agentes comerciales posee más o menos conocimientos precisos sobre una determinada transacción económica. Mediante un complejo aparato matemático este tipo de propuestas simulan operaciones comerciales donde, por ejemplo, una parte es inconsciente de las deudas que trae consigo una determinada empresa, mientras que la otra parte es inconsciente de los beneficios que determinadas acciones de dicha empresa van a generar. Los posibles resultados de una transacción económica dependerán, por tanto, del estado de conciencia o inconciencia de los agentes implicados y este tipo de propuestas lógicas son capaces de simular los resultados de algunas operaciones económicas.

A nivel cronológico cabría destacar como primer acercamiento el texto de (Modica y Rustichini, 1999), que posteriormente fue analizado por uno de los creadores de la lógica de la conciencia en (Halpern, 2001), mostrando que esa propuesta se deja reducir al sistema original de la lógica de la conciencia. Aunque esto sea así, la vía iniciada por Modica y Rustichini ha tenido bastante éxito. Se pueden resaltar, además del artículo ya mencionado, los artículos (Halpern y Rego, 2008), (Heifetz et. al., 2008) y (Heifetz et. al., 2013), todos ellos en la misma línea que los anteriores.

Una vía distinta de desarrollo dentro de la lógica epistémica, aunque más cercana a los trabajos aplicados a la economía que a los más filosóficos, viene representada por G. Sillari en (Sillari, 2007) y (Sillari, 2008). En el primero de estos trabajos, de carácter más teórico, salvo la última parte, dedicada a una lógica de la conciencia de primer orden, el autor analiza tres modelos en los que (también) cabe incorporar la conciencia y afirma que en el origen de la lógica epistémica (Hintikka, 1962) se encuentra ya el germen de lo que luego en (Fagin y Halpern, 1988) se desarrolló como lógica de la conciencia. El segundo de los trabajos de Sillari en esta dirección es un estudio comparativo de la propuesta del propio Hintikka para solventar la omnisciencia lógica, los mundos imposibles, frente a una lógica de la conciencia de primer orden (ampliando la que el autor plantea en su trabajo del año anterior). El resultado de este trabajo es la prueba de que ambos sistemas, bajo las condiciones concretas en las que los define el autor, tienen la misma expresividad lógica, esto es, son capaces de formalizar el mismo tipo de estructuras lógicas.

\section{CONCLUSIONES}

La lógica de la conciencia es, por un lado, una rama más de la lógica epistémica contemporánea y, por otro lado, una rama filosófica y epistemológicamente muy peculiar, pues representa la formalización de muchas de las intuiciones más básicas en torno a la formación y modificación del conocimiento en los agentes epistémicos por excelencia, los seres humanos. Aunque hasta la fecha 
se ha escrito poco sobre esto, es un hecho que subyace a la mayoría de los textos reseñados (y muchos de los no incluidos aquí).

A modo de conclusión se puede decir que la lógica de la conciencia, que se inició como una alternativa más que solventa el problema de la omnisciencia lógica, ha experimentado un notable crecimiento, siendo incorporada a muchos otros enfoques lógicos y teniendo, además, algunas aplicaciones prácticas muy útiles en el ámbito de la teoría de juegos o la economía. Todo ello supone una muestra del gran potencial que entraña esta lógica que, en la actualidad, se encuentra insertada (aunque con una amplia diversidad terminológica) en una gran parte de los nuevos desarrollos dinámicos de la lógica epistémica.

\section{REFERENCIAS BIBLIOGRÁFICAS}

$\AA ̊$ ANOTES, T. y ALECHINA, N. (2014). Journal of Logic, Language and Information, 23: 105.

ARTEMOV, S. (2008). «The logic of justification». The Review of Symbolic Logic, 1(4): 477-513.

ARTEMOV, S. (2018). «Justification Awareness Models». En ARTEMOV, S. y NERODE, A. (eds.): Logical Foundations of Computer Science. LFCS 2018. Lecture Notes in Computer Science, 22-36.

ARTEMOV, S. N. y NOGINA, E. (2005). «Introducing justification into epistemic logic». Journal of Logic and Computation, 15(6): 1059-1073.

BALTAG, A., RENNE, B. y SMETS, S. (2014). «The logic of justified belief, explicit knowledge, and conclusive evidence», Annals of Pure and Applied Logic, Volume 165, Issue 1, 49-81.

BURRIEZA, A. y FERNÁNDEZ-FERNÁNDEZ, C. (2015). «Distributed explicit knowledge and collective awareness». En Bulletin of the European Association for Theoretical Computer Science, Issue 116, 195-203.

BURRIEZA, A. y YUSTE-GINEL, A. (2019). «Argument Evaluation in Multi-agent Justification Logics», Logic Journal of the IGPL. (En prensa, accesible tras publicación en DOI: 10.1093/jigpal/jzz046).

FAGIN, R., HALPERN, J., MOSES, Y. y VARDI, M. Y. (1995). Reasoning about knowledge. Cambridge, Mass: The MIT Press.

FAGIN, R. y HALPERN, J. Y. (1988). «Belief, awareness, and limited reasoning». Artificial Intelligence, 34(1): 39-76.

FERNÁNDEZ-FERNÁNDEZ，C. y VELÁZQUEZ-QUESADA，F. R. (2018). «Reconsidering the 'ingredients' of explicit knowledge». En ARAZIM, P. y LÁVICKA, T., editors, The Logica Yearbook 2017, 47-60. Londres: College Publications.

FERNÁNDEZ-FERNÁNDEZ, C. y VELÁZQUEZ-QUESADA, F. R. (2019a). «A Formal Model for Explicit Knowledge as Awareness-Of Plus AwarenessThat». En SEDLÁR, I. y BLICHA, M., editors, The Logica Yearbook 2018, 101-115. Londres: College Publications. 
FERNÁNDEZ-FERNÁNDEZ，C. y VELÁZQUEZ-QUESADA， F. R. (2019b). «Awareness of and awareness that: their combination and dynamics», Logic Journal of the IGPL. (En prensa, accesible tras publicación en DOI: 10.1093/jigpal/jzz043)

GROSSI, D. y VELÁZQUEZ-QUESADA, F. R. (2010). «Twelve angry men: a dynamic-epistemic study of awareness, implicit and explicit information». En GROSSI D, KURZEN L, VELÁZQUEZ-QUESADA FR (eds), Logic and interactive rationality. Seminar's yearbook 2009. ILLC, The Netherlands, 42-68.

GROSSI, D. y VELÁZQUEZ-QUESADA, F. R. (2015). «Syntactic awareness in logical dynamics». Synthese, 192(12): 4071-4105.

HALPERN, J. Y. (2001). «Alternative Semantics for Unawareness». Games and Economic Behavior, 37, 321-339.

HALPERN, J. Y. y REGG, L. C. (2008). «Interactive unawareness revisited». Games and Economic Behavior, 62(1): 232-262.

HEIFETZ, A., MEIER, M. y SCHIPPER, B. C. (2006). «Interactive unawareness». Journal of Economic Theory, 130(1): 78-94.

HEIFETZ, A., MEIER, M. y SCHIPPER, B. C. (2008). «A canonical model for interactive unawareness». Games and Economic Behavior, 62(1): 304-324.

HEIFETZ, A., MEIER, M., y SCHIPPER, B. (2013). «Dynamic unawareness and rationalizable behavior». Games and Economic Behavior, 81(1): 50-68.

HILL, B. (2010). «Awareness dynamics». Journal of Philosophical Logic, 39(2): 113 137.

HINTIKKA, J. (1962). Knowledge and Belief. Ithaca: Cornell U.P.

HINTIKKA, J. (1975). «Impossible possible worlds vindicated». Journal of Philosophical Logic, 475-484.

HUANG, Z. y KWAST, K. (1991). «Awareness, negation and Logical omniscience». En VAN EIJCK, J. (ed), Logics in AI. JELIA 1990. Lecture Notes in Computer Science, vol. 478, 282-300.

KONOLIGE, K. (1986). «What awareness isn't: A sentential view of implicit and explicit belief». En HALPERN, J. Y., editor, Proceedings of the 1st Conference on Theoretical Aspects of Reasoning about Knowledge, Monterey, CA, March 1986, 241-250.

MEYER, J.-J. CH. y VAN DER HOEK, W. (1995). Epistemic Logic for AI and Computer Science. Cambridge: Cambridge University Press.

MODICA, S. y RUSTICHINI, A. (1999). «Unawareness and Partitional Information Structures», Games and Economic Behavior, Volume 27, Issue 2, 265-298.

MURILLO-CORCHADO, M. V. y NEPOMUCENO-FERNÁNDEZ, Á. (2019). «Giro dinámico y lógica de la investigación científica». Revista de Humanidades de Valparaíso, 13, 68-89.

SCHIPPER, B. C. (2015). «Awareness». En VAN DITMARSCH, H., HALPERN, J. Y., VAN DER HOEK, W. y KOOI, B., editors, Handbook of Epistemic Logic. Londres: College Publications, 77-146. 
SEDLÁR, I. (2013). «Justifications, awareness and epistemic dynamics». En ARTEMOV, S. N. y NERODE, A., editors, Logical Foundations of Computer Science, International Symposium, LFCS 2013, San Diego, CA, USA, January 6-8, 2013. Proceedings, volume 7734 of Lecture Notes in Computer Science, 307-318.

SILLARI, G. (2007). «Models of Awareness». En BONANNO, G., VAN DER HOEK, W. y WOOLDRIDGE, M. (editors), Logic and the Foundations of Game and Decision Theory, Volume 2 of Texts in Logic and Games. Amsterdam University Press, 209-240.

SILLARI, G. (2008). «Quantified Logic of Awareness and Impossible Worlds». The Review of Symbolic Logic, 1(4): 514-529.

VAN BENTHEM, J. (2011). Logical Dynamics of Information and Interaction. Cambridge: Cambridge University Press.

VAN BENTHEM, J. y VELÁZQUEZ-QUESADA, F. R. (2010). «The dynamics of awareness». Synthese, 177 (Supplement-1): 5-27.

VAN DITMARSCH, H. y FRENCH, T. (2009). «Awareness and Forgetting of Facts and Agents». IEEE/WIC/ACM International Joint Conference on Web Intelligence and Intelligent Agent Technology, Milan, Italy, 478-483.

VAN DITMARSCH, H. y FRENCH, T. (2011). «Becoming aware of propositional variables». En BANERJEE, M. y SETH, A., editors, Logic and Its Applications - 4th Indian Conference, ICLA 2011, Delhi, India, January 5-11, 2011. Proceedings, volume 6521 of Lecture Notes in Computer Science, 204-218.

VAN DITMARSCH, H., FRENCH, T. y VELÁZQUEZ-QUESADA, F. R. (2012). «Action models for knowledge and awareness». En VAN DER HOEK, W., PADGHAM, L., CONITZER, V. y WINIKO, M., editors, AAMAS 2012, Valencia, Spain, June 4-8, 1091-1098.

VAN DITMARSCH, H., FRENCH, T., VELÁZQUEZ-QUESADA, F. R. y WÁNG, Y. N. (2013). «Knowledge, Awareness, and Bisimulation». En TARK 2013 - Proceedings of the 14th Conference on Theoretical Aspects of Rationality and Knowledge, Vol. 1, 61-70.

VAN DITMARSCH, H., FRENCH, T., VELÁZQUEZ-QUESADA, F. R. y WÁNG, Y. N. (2018). «Implicit, explicit and speculative knowledge», Artificial Intelligence, Volume 256, 35-67.

VAN DITMARSCH, H., VAN DER HOEK, W. y KOOI, B. (2008). Dynamic Epistemic Logic. Dordrecht: Springer.

VELÁZQUEZ-QUESADA, F. R. (2011). Small steps in dynamics of information. $P h D$ thesis, Institute for Logic, Language and Computation. Amsterdam: Universiteit van Amsterdam.

VELÁZQUEZ-QUESADA, F. R. (2013). «Explicit and implicit knowledge in neighbourhood models». En GROSSI, D., ROY, O. y HUANG, H., editors, Logic, Rationality, and Interaction - 4th International Workshop, LORI 2013, Hangzhou, China, October 9-12, Proceedings, volume 8196 of Lecture Notes in Computer Science, 239-252. 
VELÁZQUEZ-QUESADA, F. R. (2014). «Dynamic epistemic logic for implicit and explicit beliefs». Journal of Logic, Language and Information, 23(2): 107140.

VELÁZQUEZ-QUESADA， F. R. (2018). «Bisimulation characterization and expressivity hierarchy of languages for epistemic awareness models», Journal of Logic and Computation, Volume 28, Issue 8, December 2018, 1805-1832.

VON WRIGHT, G. H. (1951). An Essay in Modal Logic. Amsterdam: North-Holland.

Claudia FernándeZ-FERnÁNDEZ es doctora en filosofía y profesora sustituta interina del Área de Lógica y Filosofía de la Ciencia del Departamento de Filosofía de la Universidad de Málaga.

Lineas de Investigación:

Filosofía de la Lógica, Lógica epistémica, Epistemología contemporánea

Publicaciones recientes:

FERNÁNDEZ-FERNÁNDEZ, C. y VELÁZQUEZ-QUESADA, F. R. (2019a). «A Formal Model for Explicit Knowledge as Awareness-Of Plus AwarenessThat». En SEDLÁR, I. y BLICHA, M., editors, The Logica Yearbook 2018, 101-115. Londres: College Publications.

FERNÁNDEZ-FERNÁNDEZ, C. y VELÁZQUEZ-QUESADA, F. R. (2019b). «Awareness of and awareness that: their combination and dynamics», Logic Journal of the IGPL. (En prensa, accesible tras publicación en DOI: 10.1093/jigpal/jzz043)

Correo electrónico: cffernandez@uma.es 